\title{
The Relationship between Energy-Adjusted Dietary Inflammatory Index (E-DII) with Quality of Life and Inflammatory Markers among Overweight and Obese Iranian Women
}

\section{Nasim Ghodoosi}

Tehran University of Medical Sciences

Hana Arghavani

Tehran University of Medical Sciences

Atieh Mirzababaei

Tehran University of Medical Sciences

Mir Saeed Yekaninejad

Tehran University of Medical Sciences

\section{Seyed Ali Keshavarz}

Tehran University of Medical Sciences

Hossein Imani

Tehran University of Medical Sciences

Khadijeh - mirzaei ( $\square$ mirzaei_kh@tums.ac.ir)

dr https://orcid.org/0000-0003-3631-7723

\section{Research}

Keywords: Energy-Adjusted dietary inflammatory index, overweight, Obesity, Quality of life, hs-CRP

Posted Date: July 28th, 2020

DOI: https://doi.org/10.21203/rs.3.rs-47452/v1

License: (c) (1) This work is licensed under a Creative Commons Attribution 4.0 International License. Read Full License 


\section{Abstract \\ Background}

Overweight and obesity are associated with higher levels of inflammatory markers, inflammatory diseases, and lower quality of life. Recent investigations have shown that diet can modify these complications and improve quality of life.

\section{Objective}

The objective of this study was to investigate associations between Energy-Adjusted Dietary Inflammatory Index (EDII) with quality of life and serum inflammatory markers.

\section{Methods}

In the current cross-sectional study, a total of 280 females from health centers in Tehran, Iran (2018) were selected for the study using a multistage cluster random sampling method. The EDII was computed based on dietary intake, assessed by a previously validated 147-item food frequency questionnaire (FFQ). Before calculating EDII, a residual method was used to adjust energy intake. SF-36 questionnaires were used for evaluating quality of life. Concentrations of high-sensitivity C-reactive protein (hs-CRP) were determined using collected serum samples.

\section{Results}

The mean age of the study participants was $36.30 \pm 8.05$ years. Analyses were performed using multivariable linear regression, adjusting for age, weight, physical activity, smoking status, economic status and employment status. Linear regression analysis demonstrated that E-DII were significantly associated with certain quality of life criteria, such as physical function, mental health, and vitality $(\beta=$ $5.58,95 \% \mathrm{Cl} 0.72,10.43, p=0.024, \beta=16.88,95 \% \mathrm{Cl} 10.75,23, p<0.0001$ and $\beta=14.29,95 \% \mathrm{Cl} 9.48$, $20.36, p<0.0001$, respectively). No significant associations were observed between EDII and serum level hs-CRP.

\section{Conclusion}

It was found that dietary inflammatory potential decreased some quality of life measurements and levels in overweight and obese Iranian women.

\section{Background}


Overweight and obesity is excessive fat accumulation, defined as BMI (25-29.9) and BMI $\geq 30$ respectively, according to WHO definitions $[1,2]$. The worldwide prevalence of overweight and obesity in developed and developing countries has increased substantially in recent decades [3]. Overweight and obesity are common in Iran, and are significantly more prevalent among women than men [4]. Accumulating evidence indicates that obesity is closely associated with an increased risk of cardiovascular disease (CVD), hypertension (HTN), type 2 diabetes mellitus (T2DM), hyperlipidemia, strokes, certain cancers, sleep apnea, liver and gall bladder disease, osteoarthritis and poor health quality of life (HQOL) [5].

In addition to medical complications, overweight and obesity is accompanied by a decline in healthrelated quality of life factors, such as physical functioning, psychosocial functioning, and emotional wellbeing [6-9]. The results of recent studies shows that overweight and obese people with lower BMI have higher quality of life (QoL) than those with higher BMI scores [10]. The rising prevalence and healthrelated consequences of overweight and obesity make it a public health concern all over the world $[3,11]$. The concept of quality of life is a complex, multifaceted construct that includes various aspects, such as physical health and psychological health [12].

Obesity is typically associated with a chronic state of systemic low-grade inflammation, since adipocytes result in the expression of cytokines such as hs-CRP $[13,14]$. Recent studies have shown reliable associations between diet and systemic inflammation. Additionally, it has also been reported that diet can play a major and significant role in quality of life. Previous research has shown that adherence to an anti-inflammatory diet was significantly associated with better health-related quality of life $[15,16]$. The energy-adjusted dietary inflammatory index is a validated method developed to characterize dietary inflammatory potential [17]. In view of all the above, it was hypothesized that greater adherence to an anti-inflammatory diet would be associated with lower low-grade inflammation and greater quality of life in overweight and obese patients. To the researchers' knowledge, this is the first study investigating the relationship between energy-adjusted dietary inflammatory index and quality of life.

\section{Methods}

\section{Study Design and Participants}

The current cross-sectional study was conducted among overweight and obese women who attended health centers in Tehran, Iran, in 2018. A random sample of 280 women was selected from 20 various health centers by a multistage cluster random sampling method. Eligible criteria included body mass index in the range of $25-40 \mathrm{~kg} / \mathrm{m}^{2}$. Exclusion criteria included: history of cardiovascular disease, hypertension, diabetes mellitus, hepatic or renal disease, alcohol usage, medicine usage other than birth control pills, pregnancy or lactation, following a specific diet or body weight fluctuation over the past 1 year. Informed consent was obtained from all participants. This study was approved by the research council (research project number: 95-03-161-33142, 95-04-161-33893) and ethics committee (research ethics number: IR.TUMS.VCR.REC.1395.1597). 


\section{Dietary Assessment}

Participants' dietary intake over the past year was assessed using a valid and reliable semi-quantitative food frequency questionnaire (FFQ). This FFQ consists of 147 food items with standard serving sizes, and participants were asked to specify their consumption frequency for each food item on a daily, weekly, monthly or yearly basis. Then, nutrient and energy intakes were computed using NUTRITIONIST IV software (version 7.0; N-Squared Computing, Salem, OR), which was tailored for Iranian foods. For calculating EDII, all nutrient values were adjusted for energy intake using the residual method.

\section{Dietary Inflammatory Index Calculation}

To calculate EDII for the participants of this study, the dietary data were first linked to the regionally representative world database, which provided a robust estimate of a mean and standard deviation for each parameter. These then become the multipliers to express an individual's exposure relative to the 'standard global mean' as a z-score. A z-score for each food consumed was calculated by subtracting the 'standard mean' from the actual food parameter value, and divided by its standard deviation. Next, to minimize the effect of 'right skewing', this value was then converted to a centered percentile score, which was then multiplied by the respective food parameter inflammatory effect score to obtain the subject's food parameter-specific EDII score. All of the food-parameter-specific EDII scores were then summed together to create an overall EDII score for every subject in the study [18]. In total, the EDII computed based on this study's FFQ includes data on 29 of the 45 possible food variables composing the EDII: energy, carbohydrate, protein, fat, fiber, cholesterol, trans fat, SFAs, MUFAs, PUFAs, omega-3, omega-6, niacin, thiamin, riboflavin, vitamin B-6, vitamin B-12, iron, magnesium, selenium, zinc, vitamin A, vitamin $C$, vitamin $D$, vitamin $E$, folic acid, b-carotene, caffeine, onion and tea.

\section{Quality of Life Assessment}

The SF-36 is a short-form, self-administered quality of life scoring questionnaire. It consists of 36 questions, 35 of which are compressed into eight multi-item scales including: physical functioning (PF), role-physical (RP), bodily pain (BP), general health (GH), vitality (VT), social functioning (SF), role emotional (RE), and mental health (MH). (1) Physical Functioning (PF) is a 10-question scale that captures abilities to deal with the physical requirement of life, such as attending to personal needs, walking, and flexibility. (2) Role-Physical (RP) is a four-item scale that evaluates the extent to which physical capabilities limit activity. (3) Bodily Pain (BP) is a two-item scale that evaluates the perceived amount of pain experienced during the most recent 4 weeks and the extent to which that pain interfered with normal work activities. (4) General Health $(\mathrm{GH})$ is a five-item scale that evaluates general health in terms of personal perception. (5) Vitality (VT) is a four-item scale that evaluates feeling of pep, energy, and fatigue. (6) Social Functioning (SF) is a two item scale that evaluates the extent and amount of time, if any, that physical health or emotional problems interfered with family, friends, and other social interactions during the most recent 4 weeks. (7) Role-Emotional (RE) is a three item scale that evaluates the extent, if any, to which emotional factors interfere with work or other activities. (8) Mental Health (MH) is a five-item scale that evaluates feelings principally of anxiety and depression $[19,20]$. The SF-36 also 
includes a question self-evaluating health changes in the past year (reported health), which does not belong to the eight dimensions, or the total SF-36 score. Each of these 8 dimensions has a score between 0 (worst health) to 100 (best health). [21-23]

\section{Biochemical Assessment:}

Blood samples were collected early in the morning after 12-hour overnight fasting. Serum was separated from whole blood samples and stored at $-80^{\circ} \mathrm{C}$ until the assay Serum hs-CRP levels was measured by an immunoturbidimetric assay with a Pars Azmoon kit (Pars Azmoon Inc. Tehran, Iran).

\section{Anthropometric Assessment:}

Anthropometric measures, including body weight, body mass index, waist circumferences and waist-hip ratio, were measured in an overnight fasting state, without shoes, with minimal clothing and by the use of a multi-frequency bioelectrical impedance analyzer In-body 770 scanner (In-body Co., Seoul, Korea). Height was measured with a Seca 206 scale, based on standard protocol.

\section{Assessment of Other Variables:}

Physical activity status and socio-demographic information was obtained by questionnaire. Data on physical activity was gathered using the IPAQ questionnaire. Activity was classified as light, medium or heavy levels (IPAQ). The metabolic equation hours per day score (MET-min/week) was then calculated for each subject $[24,25]$.

\section{Statistical Analyses:}

The EDII was analyzed as a dichotomous variable, categorized based on the median value of the EDII (0.05). EDII (as dichotomous) was examined across the following characteristics: age, weight, height, economic status, BMI, waist circumference, waist-hip ratio, energy intake, physical activity, and quality of life measurements, via independent sample T-test analyses. Comparisons of different food group intakes across the EDII quartiles were analyzed through an independent sample T-test. Multivariable linear regression analyses of the continuous EDII score were conducted to determine the association of the EDII with quality of life and hs-CRP levels. Variables were adjusted for the following confounding factors: age, weight, physical activity, smoking, economic status and employment status. The results are reported as percentage change $(\beta)$ with $95 \%$ confidence intervals $(95 \% \mathrm{Cl})$. Statistical analysis was performed using SPSS (version 21) (SPSS Inc., Chicago, USA). Significance was set at a probability of $\leq 0.05$ for all tests.

\section{Results}

The dietary inflammatory potential scores in this study, as measured by EDII, ranged from -4.14 (most anti-inflammatory score) to 3.89 (most pro-inflammatory score). The mean (SD) age of the participants at recruitment was 36 (8). Table 1 presents participants' characteristics in relation to different categories of dietary inflammatory indices. EDII was categorized into anti-inflammatory (EDII $\leq 0 / 05)$ and pro- 
inflammatory (EDII > 0/06) diets, based on the median value (0.05). When EDII was converted into two groups, significant differences were observed for IPAC $(P<0.0001)$. The hs-CRP level showed no significant change in the higher EDII score group compared to the other group (PC: $4 / 26 \pm 4 / 42 \%$ vs. $4 / 07 \pm 4 / 34 \% ; P=0.856)$.

Significant inverse differences were found between the three dimensions of SF-36, including physical functioning $(P=0.033)$, mental health $(P=0.021)$ and vitality $(P=0.031)$, with anti-inflammatory and proinflammatory diet groups. For the other five dimensions, such as general health, role-physical, role emotional, social functioning, bodily pain and health transition, decreasing trends were observed across EDIl categories, but these relationships were not significant (Table 2).

Table 3 shows the distribution of 11 food groups: grains, refined grains, dairy products, beans, vegetables, fruits, red meat, processed meat, white meat, nut/olive group and sweet/fat group across the EDII groups. The results showed that servings of vegetables and beans $(P<0.0001)$ decreased significantly in the higher dietary inflammatory index group, whereas servings of sweet/fat group $(P<0.0001)$ increased significantly in this group.

A direct association between EDII and physical functioning levels was observed after adjustment for potential confounders, such as age, weight, smoking, economic status and employment status $(P=0.024)$ (Table 4).

Multivariable linear regression analysis with adjustments for potential confounders demonstrated that EDII were significantly associated with mental health and vitality $(\beta=5.58,95 \% \mathrm{Cl} 0.72,10.43, p=$ 0.024(Table2), $\beta=16.88,95 \% \mathrm{Cl} 10.75,23, p<0.0001$ (Table5) and $\beta=14.29,95 \% \mathrm{Cl} 9.48,20.36, p<$ 0.0001 (Table6)), respectively.

\section{Discussion:}

It is important to note that this is the first study to examine the association between dietary inflammatory potential, quality of life and inflammation levels among women. This cross-sectional study of Iranian women showed evidence of a positive association between higher (i.e. more pro-inflammatory) EDII scores with lower quality of life in certain measurements, such as physical functioning, mental health and vitality. These findings emphasize the importance of addressing overall dietary quality in future community- or population-based programs or policies to prevent chronic disease.

Some prior research has shown that inflammatory diet plays an important role in psychological health. Almudena Sánchez-Villegas et al. also determined that a pro-inflammatory diet was associated with a significantly higher risk of depression in a Mediterranean population, particularly in older subjects [26]. Tasnime N. Akbaraly et al. found that a pro-inflammatory diet was associated with recurrent depression in women, which seems not to be driven by circulating inflammatory markers [27]. There is considerable evidence that has suggested a better quality of diet or anti-inflammatory diet is associated with better quality of life and better mental health. Ujué Fresán et al. have found that a Mediterranean diet was 
associated with reduced depression risks [28]. A recent study in Australia demonstrated that better dietary quality in older adults was related with better health-related quality of life and emotional wellbeing in women [29]. The current findings are supported by an Australian study which reported that adherence to an MD pattern is associated with better mental and physical health, and that this association is particularly stronger in terms of mental health than physical health [30]. A possible mechanism seems to related to the fact that an anti-inflammatory diet is rich in nutrients such as vitamins, minerals, antioxidants, and fiber, which have beneficial health effects that have been widely demonstrated.

The results revealed no relationship between EDII and hs-CRP levels in women. To the best of the researchers' knowledge, previous studies of dietary inflammatory indices and serum hs-CRP levels are generally consistent with the findings of the present study. A number of other studies have suggested that a pro-inflammatory diet is associated with higher levels of inflammatory markers. Previous studies have shown that DII was associated with an increase in the odds of elevated hs-CRP levels (.3 mg/l) [31]. Another study in the USA demonstrated that higher DII scores were associated with inflammatory biomarkers including IL-6, TNF- $a$ and hs-CRP [32]. In the Asklepios study, no significant associations were observed between DII and inflammatory markers CRP and fibrinogen, but significant positive associations between DII and inflammatory markers IL-6 and homocysteine were observed [33].

\section{Conclusion:}

The most interesting finding of this study is that the multivariable linear regression analysis has revealed that more anti-inflammatory diets are significantly associated with higher physical function, mental health and vitality in overweight and obese women. Thus, encouraging the intake of more antiinflammatory dietary factors, such as plant-based foods rich in fiber and phytochemicals, and reducing intake of pro-inflammatory factors, such as fried foods or processed foods rich in saturated fat, may be a beneficial strategy for better physical and mental health.

The present study had some limitations that should be considered. First of all, the use of self-reported food-frequency questionnaires (FFQ) is known to contain a certain degree of measurement error, which might affect results. Moreover, an FFQ consisting of 29 instead of 45 food parameters was used for calculating EDII. The small sample size is another limitation, and data from larger sample size studies are more credible. In addition, casual relationship between energy-adjusted dietary inflammatory index, inflammatory markers, and quality of life cannot be inferred using retrospective observational studies; conducting prospective studies is thus highly recommended.

\section{Abbreviations}

BMI: Body mass index, CVD: Cardiovascular disease, C-reactive protein, EDII: Energy-Adjusted dietary inflammatory index, ELISA: Enzyme-linked immunosorbent assay, FFQ: Food frequency questionnaire, HQOL: Health quality of life, HTN: Hypertension ,Hs-CRP: High-sensitivity C-reactive protein, IL-6: Interleukin-6, IPAQ: International Physical Activity Questionnaires, MUFA: Monounsaturated fatty acids 
,METs: Metabolic Equivalent Tasks, PUFA: Polyunsaturated fatty acids, QOL: Quality of life, SFA: Saturated fatty acids, T2DM: Type 2 diabetes mellitus, TNFa: Tumor necrosis factor-a, WHO: World Health Organization

\section{Declarations}

\section{Authors' contributions}

The project was designed and implemented by NGH and KhM. Data were analyzed and interpreted NGH, HA, AM, SY, SAK and HI prepared the manuscript. KhM, supervised overall project. All authors read and approved the fnal manuscript.

\section{Acknowledgements}

We thank the School of Public Health, Tehran University of Medical Sciences and we would like to thank all the participants for their cooperation.

\section{Competing interests}

The authors declare that they have no competing interests.

\section{Availability of data and materials}

The data that support the fndings of this study are available from Khadijeh Mirzaei but restrictions apply to the availability of these data, which were used under license for the current study, and so are not publicly available. Data are however available from the authors upon reasonable request and with permission of Khadijeh Mirzae

\section{Consent for publication}

Each participant was completely informed about the study protocol and provided a written and informed consent form before taking part in the study.

\section{Ethics approval and consent to participate}

The study protocol has approved by the ethics committee of Endocrinology and Metabolism Research Center of Tehran University of Medical Sciences (TUMS) with the following identifcation: IR.TUMS.VCR.REC.1395.1597.

\section{Funding}

This study was supported by the Osteoporosis Research Center, Endocrine Diseases and Metabolism Research Institute, Tehran University of Medical Sciences, and by Grants from Tehran University of Medical Sciences (research project number: 95-03-161-33142, 95-04-161-33893) and ethics committee (research ethics number: IR.TUMS.VCR.REC.1395.1597). 


\section{References}

1. Gallus S, et al. Overweight and obesity prevalence and determinants in Italy: an update to 2010 . Eur J Nutr. 2013;52(2):677-85.

2. Pietrzyk L, et al. Obesity and obese-related chronic low-grade inflammation in promotion of colorectal cancer development. Asian Pac J Cancer Prev. 2015;16(10):4161-8.

3. Kelly T, et al. Global burden of obesity in 2005 and projections to 2030. Int J Obes. 2008;32(9):1431.

4. Ayatollahi S, Ghoreshizadeh Z. Prevalence of obesity and overweight among adults in Iran. Obesity reviews. 2010;11(5):335-7.

5. Williams EP, et al. Overweight and obesity: prevalence, consequences, and causes of a growing public health problem. Current obesity reports. 2015;4(3):363-70.

6. Kushner RF, Foster GD. Obesity and quality of life. Nutrition. 2000;16(10):947-52.

7. Fontaine KR, Barofsky I. Obesity and health-related quality of life. Obes Rev. 2001;2(3):173-82.

8. Kolotkin RL, Meter K, Williams GR. Quality of life and obesity. Obes Rev. 2001;2(4):219-29.

9. Sullivan M, et al. Why quality of life measures should be used in the treatment of patients with obesity. In: International Textbook of Obesity. New York: John Wiley \& Sons; 2001. pp. 485-510.

10. Pimenta FB, et al., The relationship between obesity and quality of life in Brazilian adults. Frontiers in psychology, 2015. 6.

11. $\mathrm{Ng} \mathrm{M}$, et al. Global, regional, and national prevalence of overweight and obesity in children and adults during 1980-2013: a systematic analysis for the Global Burden of Disease Study 2013. The lancet. 2014;384(9945):766-81.

12. Taylor $\mathrm{VH}$, et al., The impact of obesity on quality of life. Best practice \& research Clinical endocrinology \& metabolism, 2013. 27(2): p. 139-146.

13. Kim C, et al. Circulating levels of MCP-1 and IL-8 are elevated in human obese subjects and associated with obesity-related parameters. Int J Obes. 2006;30(9):1347.

14. Singer K, Lumeng CN. The initiation of metabolic inflammation in childhood obesity. J Clin Investig. 2017;127(1):65-73.

15. Costarelli V, Koretsi E, Georgitsogianni E. Health-related quality of life of Greek adolescents: the role of the Mediterranean diet. Quality of life research. 2013;22(5):951-6.

16. Veronese $\mathrm{N}$, et al. Adherence to the Mediterranean diet is associated with better quality of life: data from the Osteoarthritis Initiative. Am J Clin Nutr. 2016;104(5):1403-9.

17. Wirth $\mathrm{M}$, et al. Association of a Dietary Inflammatory Index with Inflammatory Indices and the Metabolic Syndrome among Police Officers. Journal of occupational environmental medicine/American College of Occupational Environmental Medicine. 2014;56(9):986.

18. Shivappa N, et al. Designing and developing a literature-derived, population-based dietary inflammatory index. Public Health Nutr. 2014;17(8):1689-96. 
19. McHorney CA, Ware JE Jr, Raczek AE, The MOS 36-Item Short-Form Health Survey (SF-36): II. Psychometric and clinical tests of validity in measuring physical and mental health constructs. Medical care, 1993: p. 247-263.

20. Kalantar-Zadeh K, et al. Association among SF36 quality of life measures and nutrition, hospitalization, and mortality in hemodialysis. J Am Soc Nephrol. 2001;12(12):2797-806.

21. Brazier JE, et al. Validating the SF-36 health survey questionnaire: new outcome measure for primary care. Bmj. 1992;305(6846):160-4.

22. Arefnasab Z, et al. Effect of mindfulness based stress reduction on quality of life (SF-36) and spirometry parameters, in chemically pulmonary injured veterans. Iranian journal of public health. 2013;42(9):1026.

23. Lins L, Carvalho FM, SF-36 total score as a single measure of health-related quality of life: Scoping review. SAGE open medicine, 2016. 4: p. 2050312116671725.

24. Craig $\mathrm{CL}$, et al. International physical activity questionnaire: 12-country reliability and validity. Med Sci sports Exerc. 2003;35(8):1381-95.

25. Committee IR. Guidelines for data processing and analysis of the International Physical Activity Questionnaire (IPAQ)-short and long forms. http://www. ipaq. ki. se/scoring. pdf, 2005.

26. Sánchez-Villegas A, et al. Dietary inflammatory index, cardiometabolic conditions and depression in the Seguimiento Universidad de Navarra cohort study. Br J Nutr. 2015;114(9):1471-9.

27. Akbaraly TN, et al. Dietary inflammatory index and recurrence of depressive symptoms: Results from the Whitehall II Study. Clinical Psychological Science. 2016;4(6):1125-34.

28. Fresán U, et al., Does the MIND diet decrease depression risk? A comparison with Mediterranean diet in the SUN cohort. European journal of nutrition, 2018: p. 1-12.

29. Milte CM, et al. Associations of diet quality with health-related quality of life in older Australian men and women. Experimental gerontology. 2015;64:8-16.

30. Bonaccio $\mathrm{M}$, et al. Adherence to a Mediterranean diet is associated with a better health-related quality of life: a possible role of high dietary antioxidant content. BMJ open. 2013;3(8):e003003.

31. Shivappa N, et al. A population-based dietary inflammatory index predicts levels of C-reactive protein in the Seasonal Variation of Blood Cholesterol Study (SEASONS). Public Health Nutr. 2014;17(8):1825-33.

32. Tabung FK, et al. Construct validation of the dietary inflammatory index among postmenopausal women. Ann Epidemiol. 2015;25(6):398-405.

33. Shivappa N, et al. Associations between dietary inflammatory index and inflammatory markers in the Asklepios Study. Br J Nutr. 2015;113(4):665-71.

\section{Tables}

Table 1. Participant Characteristics between EDII Groups 


\begin{tabular}{|c|c|c|c|c|}
\hline Participant Characteristics & EDII Groups & Mean & Std. Deviation & $P$ value \\
\hline \multirow[t]{2}{*}{ Age(year) } & $<=.05$ & 37.60 & 7.58 & \multirow[t]{2}{*}{0.105} \\
\hline & $.06+$ & 35.03 & 8.48 & \\
\hline \multirow[t]{2}{*}{ Weight(kg) } & $<=.05$ & 81.09 & 12.56 & \multirow[t]{2}{*}{0.317} \\
\hline & $.06+$ & 80.61 & 12.09 & \\
\hline \multirow[t]{2}{*}{ Height(cm) } & $<=.05$ & 161.25 & 6.24 & \multirow[t]{2}{*}{0.217} \\
\hline & $.06+$ & 161.33 & 5.75 & \\
\hline \multirow[t]{2}{*}{$\mathrm{BMI}\left(\mathrm{kg} / \mathrm{m}^{2}\right)$} & $<=.05$ & 30.94 & 3.99 & \multirow[t]{2}{*}{0.130} \\
\hline & $.06+$ & 30.52 & 3.56 & \\
\hline \multirow[t]{2}{*}{ Waist Circumference(cm) } & $<=.05$ & 99.25 & 10.24 & \multirow[t]{2}{*}{0.391} \\
\hline & $.06+$ & 98.97 & 9.89 & \\
\hline \multirow[t]{2}{*}{ Waist-Hip Ratio } & $<=.05$ & .93 & .05 & \multirow[t]{2}{*}{0.651} \\
\hline & $.06+$ & .93 & .05 & \\
\hline \multirow[t]{2}{*}{ Energy Intake(kcal) } & $<=.05$ & 2588.82 & 707.15 & \multirow[t]{2}{*}{0.140} \\
\hline & $.06+$ & 2658.21 & 790.42 & \\
\hline \multirow[t]{2}{*}{ IPAC(MET-min/week) } & $<=.05$ & 1162.45 & 1322.88 & \multirow[t]{2}{*}{$<0.0001$} \\
\hline & $.06+$ & 773.24 & 692.04 & \\
\hline \multirow[t]{2}{*}{ Hs-CRP(mg/L) } & $<=.05$ & 4.26 & 4.42 & \multirow[t]{2}{*}{0.856} \\
\hline & $.06+$ & 4.07 & 4.34 & \\
\hline \multirow[t]{2}{*}{ SF Total } & $<=.05$ & 77.32 & 13.43 & \multirow[t]{2}{*}{0.715} \\
\hline & $.06+$ & 72.11 & 12.80 & \\
\hline
\end{tabular}

$n=280$

Data are presented as mean \pm standard deviation.

Nutrients intake adjusted for energy intake before calculating EDII

DIl values were categorized according to the median

(DII $\leq$ 0/05: Anti-inflammatory diet, DII > 0/06: pro-inflammatory diet)

Independent sample $t$ test was used for comparison of continuous variables between DII categories 
Table 2. Quality of Life Items by Level of EDII groups

\begin{tabular}{|c|c|c|c|c|}
\hline SF-36 Items & DII Groups & Mean & Std. Deviation & $P$ value \\
\hline \multirow[t]{2}{*}{ General Health } & $<=.05$ & 68.47 & 18.88 & \multirow[t]{2}{*}{0.082} \\
\hline & $.06+$ & 66.92 & 16.77 & \\
\hline \multirow[t]{2}{*}{ Physical Functioning } & $<=.05$ & 84.37 & 16.65 & \multirow[t]{2}{*}{0.033} \\
\hline & $.06+$ & 79.16 & 22.33 & \\
\hline \multirow[t]{2}{*}{ Role Physical } & $<=.05$ & 91.24 & 28.37 & \multirow[t]{2}{*}{0.094} \\
\hline & $.06+$ & 88.15 & 32.44 & \\
\hline \multirow[t]{2}{*}{ Role Emotional } & $<=.05$ & 86.78 & 33.89 & \multirow[t]{2}{*}{0.073} \\
\hline & $.06+$ & 82.89 & 37.71 & \\
\hline \multirow[t]{2}{*}{ Social Functioning } & $<=.05$ & 73.65 & 22.71 & \multirow[t]{2}{*}{0.968} \\
\hline & $.06+$ & 70.76 & 23.53 & \\
\hline \multirow[t]{2}{*}{ Bodily Pain } & $<=.05$ & 62.31 & 21.02 & \multirow[t]{2}{*}{0.871} \\
\hline & $.06+$ & 63.09 & 20.24 & \\
\hline \multirow[t]{2}{*}{ Vitality } & $<=.05$ & 76.37 & 18.35 & \multirow[t]{2}{*}{0.031} \\
\hline & $.06+$ & 60 & 22.28 & \\
\hline \multirow[t]{2}{*}{ Mental Health } & $<=.05$ & 82.33 & 20.82 & \multirow[t]{2}{*}{0.021} \\
\hline & $.06+$ & 66.28 & 24.52 & \\
\hline \multirow[t]{2}{*}{ Health Transition Item } & $<=.05$ & 51.82 & 29.34 & \multirow[t]{2}{*}{0.056} \\
\hline & $.06+$ & 46.67 & 25.70 & \\
\hline \multirow[t]{2}{*}{ SF-36 -TOTAL } & $<=.05$ & 77.32 & 13.43 & \multirow[t]{2}{*}{0.715} \\
\hline & $.06+$ & 72.11 & 12.80 & \\
\hline
\end{tabular}

$n=280$

Data are presented as mean \pm standard deviation.

Nutrients intake adjusted for energy intake before calculating EDII

EDIl values were categorized according to the median. 
(Anti-inflammatory diet: EDII $\leq$ 0/05, pro-inflammatory diet: EDII > 0/06)

Independent sample $t$ test was used for comparison of continuous quality of life measures between EDII categories

Table 3. Relationships between Servings of Food Groups across EDII Groups 


\begin{tabular}{|c|c|c|c|c|}
\hline Food Groups(g/day) & EDII Groups & Mean & Std. Deviation & $P$ value \\
\hline \multirow[t]{2}{*}{ Grains } & $<=.05$ & 458.59 & 218.60 & \multirow[t]{2}{*}{0.749} \\
\hline & $.06+$ & 507.98 & 242.73 & \\
\hline \multirow[t]{2}{*}{ Refined Grains } & $<=.05$ & 385.37 & 220.20 & \multirow[t]{2}{*}{0.274} \\
\hline & $.06+$ & 479.92 & 243.59 & \\
\hline \multirow[t]{2}{*}{ Dairy Products } & $<=.05$ & 390.78 & 217.44 & \multirow[t]{2}{*}{0.272} \\
\hline & $.06+$ & 351.84 & 270.79 & \\
\hline \multirow[t]{2}{*}{ Beans } & $<=.05$ & 57.75 & 49.82 & \multirow[t]{2}{*}{$<0.0001$} \\
\hline & $.06+$ & 41.01 & 26.91 & \\
\hline \multirow[t]{2}{*}{ Vegetables } & $<=.05$ & 535.37 & 250.44 & \multirow[t]{2}{*}{$<0.0001$} \\
\hline & $.06+$ & 292.86 & 193.06 & \\
\hline \multirow[t]{2}{*}{ Fruits } & $<=.05$ & 653.14 & 354.93 & \multirow[t]{2}{*}{0.060} \\
\hline & $.06+$ & 454.64 & 326.29 & \\
\hline \multirow[t]{2}{*}{ Red Meat } & $<=.05$ & 46.45 & 24.31 & \multirow[t]{2}{*}{0.995} \\
\hline & $.06+$ & 42.80 & 25.40 & \\
\hline \multirow[t]{2}{*}{ Processed Meat } & $<=.05$ & 4.08 & 10.48 & \multirow[t]{2}{*}{0.862} \\
\hline & $.06+$ & 5.65 & 7.92 & \\
\hline \multirow[t]{2}{*}{ White Meat } & $<=.05$ & 50.27 & 40.06 & \multirow[t]{2}{*}{0.987} \\
\hline & $.06+$ & 43.71 & 51.31 & \\
\hline \multirow[t]{2}{*}{ Nut.Olive } & $<=.05$ & 13.87 & 11.52 & \multirow[t]{2}{*}{0.116} \\
\hline & $.06+$ & 9.94 & 10.93 & \\
\hline \multirow[t]{2}{*}{ Sweet. Fat } & $<=.05$ & 80.27 & 50.13 & \multirow[t]{2}{*}{$<0.0001$} \\
\hline & $.06+$ & 126.26 & 111.59 & \\
\hline
\end{tabular}

$n=280$

Data are presented as mean \pm standard deviation.

Nutrients intake adjusted for energy intake before calculating EDII 
EDIl values were categorized according to the median.

(EDII $\leq$ 0/05: Anti-inflammatory diet, EDII > 0/06: Pro-inflammatory diet)

Independent sample $t$ test was used for comparison of food group's intake between EDII

categories

Table 4. Relationship between EDII and physical functioning

\begin{tabular}{|llll|}
\hline Variables & B & $95 \%(\mathrm{Cl})$ & P value \\
\hline Anti-inflammatory diet & 5.58 & $(0.72,10.43)$ & 0.024 \\
\hline Pro-inflammatory diet &. Ref & & .Ref \\
\hline Age (years) & 0.07 & $(-0.24,0.38)$ & 0.653 \\
\hline Weight (kg) & 0.01 & $(-0.18,0.20)$ & 0.919 \\
\hline Smoking (Smoker) & -3.52 & $(-14.02,6.98)$ & 0.511 \\
\hline Smoking (Non-smoker) &. Ref & &. Ref \\
\hline Economic status (Poor) & -0.27 & $(-7.13,6.59)$ & 0.938 \\
\hline Economic status (Medium) & 3.86 & $(-2.04,9.76)$ & 0.199 \\
\hline Economic status (Good) & .Ref & &. Ref \\
\hline Job status (Unemployed) & -1.71 & $(-3.52,6.93)$ & 0.522 \\
\hline Job status (Employed) &. Ref & &. Ref \\
\hline
\end{tabular}

$n=280$

(Anti-inflammatory diet: EDII $\leq$ 0/05, pro-inflammatory diet: EDII > 0/06)

Multivariable linear regression test was used to investigate the relationship between

EDII and physical

functioning

Table 5. Relationships between EDII and Mental Health 


\begin{tabular}{|llll|}
\hline Variables & B & $95 \%(\mathrm{Cl})$ & P value \\
\hline Anti-Inflammatory Diet & 16.88 & $(10.75,23.00)$ & $<0.0001$ \\
\hline Pro-Inflammatory Diet &. Ref & &. Ref \\
\hline Age (years) & -.14 & $(-0.52,0.25)$ & .478 \\
\hline Weight (kg) & .05 & $(-0.21,0.31)$ & .704 \\
\hline Physical Activity (MET-min/week ) & -2.12 & $(-5.12,0.87)$ & .164 \\
\hline Smoking (Smoker) & 6.59 & $(-6.82,20.01)$ & .335 \\
\hline Smoking (Non-smoker) &. Ref & &. Ref \\
\hline Economic Status (Poor) & -1.13 & $(-9.62,7.37)$ & .795 \\
\hline Economic Status (Medium) & -.98 & $(-8.24,6.28)$ & .791 \\
\hline Economic Status (Good) &. Ref & &. Ref \\
\hline Job Status (Unemployed) & 4.37 & $(-2.09,10.82)$ & .185 \\
\hline Job Status (Employed) &. Ref & &. Ref \\
\hline
\end{tabular}

$n=280$

(Anti-inflammatory diet: EDII $\leq$ 0/05, pro-inflammatory diet: EDII > 0/06)

Multivariable linear regression test was used to investigate the relationship between EDII and mental health

Table 6. Relationships between EDII and Vitality 


\begin{tabular}{|llll|}
\hline Variables & $\beta$ & $95 \%(\mathrm{Cl})$ & P value \\
\hline Anti-Inflammatory Diet & 14.92 & $(9.48,20.36)$ & $<0.0001$ \\
\hline Pro-Inflammatory Diet &. Ref & &. Ref \\
\hline Age (years) & 0.04 & $(-0.30,0.37)$ & 0.837 \\
\hline Weight (kg) & 0.01 & $(-0.22,0.23)$ & 0.961 \\
\hline Physical Activity (MET-min/week) & 2.23 & $(-0.43,4.89)$ & 0.100 \\
\hline Smoking (Smoker) & 8.02 & $(-3.49,19.54)$ & 0.172 \\
\hline Smoking (Non-smoker) &. Ref & &. Ref \\
\hline Economic Status )Poor) & -1.27 & $(-8.84,6.29)$ & 0.742 \\
\hline Economic Status (Medium) & 0.39 & $(-6.04,6.82)$ & 0.906 \\
\hline Economic Status (Good) &. Ref & &. Ref \\
\hline Job status (Unemployed) & 2.56 & $(-3.11,8.22)$ & 0.377 \\
\hline Job status (Employed) &. Ref & &. Ref \\
\hline
\end{tabular}

$n=280$

(Anti-inflammatory diet: EDII $\leq$ 0/05, pro-inflammatory diet: EDII > 0/06)

Multivariable linear regression test was used to investigate the relationship between EDII and vitality 